\title{
Simplified Overlay Architecture for Cognitive Wireless Systems
}

\author{
Monirosharieh Vameghestahbanati, Hasan S. Mir, and Mohamed El-Tarhuni
}

\begin{abstract}
In this paper, we present two simplified scenarios for interference mitigation in overlay cognitive radio systems. These schemes are based on either optimizing the secondary user transmitter-only or the secondary user receiver-only without any changes to the existing primary user system in order to minimize the secondary system mean-square error (MSE) and the excess mean-square error at the primary system. These schemes are compared to a system that jointly optimizes the transmitter and receiver for the secondary system resulting in high complexity. Analytical and simulations results for the mean-square error under different cross-talk interference levels are presented in this paper. The results show that the receiver-only optimization incurs about $5 \mathrm{~dB}$ degradation in the secondary user MSE compared to the optimal system that jointly optimizes transmitter and receiver. It is also shown the receiver-only optimization does not degrade the excess MSE performance for the primary user causing minimal increase in the interference level. On the other hand, optimizing the transmitter-only results in a slightly small improvement to the primary system excess MSE and hence reducing the interference, but it fails in providing acceptable performance for the secondary user resulting in about $25 \mathrm{~dB}$ degradation in MSE.
\end{abstract}

Index Terms-Cognitive radio (CR), crosstalk, mean square error (MSE), overlay system.

\section{INTRODUCTION}

With the increasing demands for higher data rate services, the spectral efficiency of modern communication systems should be improved as they should be able to deliver data at an increasing rate as well as a lower cost per bit [1]. On the other hand, the natural prime spectrum is scarce and cannot fulfill the requirements of such demands.

Cognitive radio (CR), a recently developed paradigm, is a low-cost alternative to the static frequency radio systems, which can alleviate the frequency scarcity problem [2], [3]. In particular, the legacy (existing) system, which is the primary user, can coexist with unlicensed overlay (secondary) system, providing that the overlay user does not degrade the performance of the legacy system [4].

To solve the spectrum sharing problem in cognitive wireless systems, [5] proposed a framework that considers the quality of service (QoS) constraints for the overlay users and the interference constraints for the legacy users. In [6], the joint design problem of precoder and receiver between

Manuscript received January 9, 2014; revised May 12, 2014

M. Vameghestahbanati is with the Department of Systems and Computer Engineering, Carleton University, PO Box K1S 5B6 Ottawa, Canada (e-mail: moniroshariehvameghe@sce.carleton.ca).

H. Mir and M. El-Tarhuni are with the Department of Electrical Engineering of American University of Sharjah, PO Box 26666 Sharjah, United Arab Emirates (e-mail:hmir@aus.edu, mtarhuni@aus.edu). transmitted signal and its estimate at the receiver in a downlink single user multiple-input-multiple-output (MIMO) system is investigated. The transceiver optimization of a MIMO CR system using mean-squared error (MSE) criterion is examined in [4], while [7] treated the transceiver optimization in a multiple-input-single-output (MISO) CR network using MSE method.

In [8], we have explored the joint transmitter/ receiver optimization for the overlay system, however, from the practical standpoint it is less complicated to fix either the overlay transmitter or receiver and to optimize the receiver or transmitter. In this paper, we study the performance loss that is incurred as a result of not performing the joint optimization.

The rest of this paper is organized as follows. Section II describes the system model and the problem formulation. Section III provides the analytical and simulation results, and Section IV presents the conclusion.

\section{PRPBLEM FORMULATION}

Fig. 1 shows the legacy and overlay system models. The inputs to the legacy and overlay transmitters are assumed to be independent and identically distributed (i.i.d) sequences: $z_{2 n} \in\{ \pm 1\}$ and $z_{1 n} \in\{ \pm 1\}$. The legacy system transmitter has an impulse response $h_{t}^{(l)}(t)$, while the overlay system transmitter has an impulse response $h_{t}^{(o)}(t)$. Furthermore, $h_{r}^{(l)}(t)$ and $h_{r}^{(o)}(t)$ represent the impulse response for legacy system and overlay system receivers, respectively. The direct path with channel impulse response $h_{c}^{(l l)}(t)$ is used by the legacy system corrupted by the additive white Gaussian noise (AWGN), $w_{2}(t)$. On the other hand, the direct channel with impulse response $h_{c}^{(o o)}(t)$ is used by the overlay system but corrupted by AWGN, $w_{1}(t)$. It is assumed the interference channel from the overlay transmitter and to the legacy receiver is represented by $h_{c}^{(o l)}(t)$, whereas the interference path from the legacy transmitter to the overlay receiver is denoted by $h_{c}^{(l o)}(t)$.

The legacy and overlay receivers process $r_{2}(t)$ and $r_{1}(t)$ to produce the output signals $x_{2}(t)$ and $x_{1}(t)$, which will be then processed by the decision devices in order to generate $\hat{z}_{2 n}$ and $\hat{z}_{1 n}$.

In this paper, we perform the receiver-only optimization and the transmitter-only optimization by optimizing only the overlay receiver filter, $h_{r}^{(o)}(t)$, and the overlay transmitter filter, $h_{t}^{(o)}(t)$ so as to allow the simultaneous operation of both the overlay and the legacy systems considering that the legacy system filters, $h_{t}^{(l)}(t)$ and $h_{r}^{(l)}(t)$, are fixed and cannot be modified. It is assumed that all channels $\left(h_{c}^{(o o)}(t)\right.$, 
$h_{c}^{(l l)}(t), h_{c}^{(o l)}(t)$, and $\left.h_{c}^{(l o)}(t)\right)$ follow complex Gaussian distribution which leads to Rayleigh fading channels.
Furthermore, the knowledge of all channels is assumed to be available through appropriate feedback mechanisms.

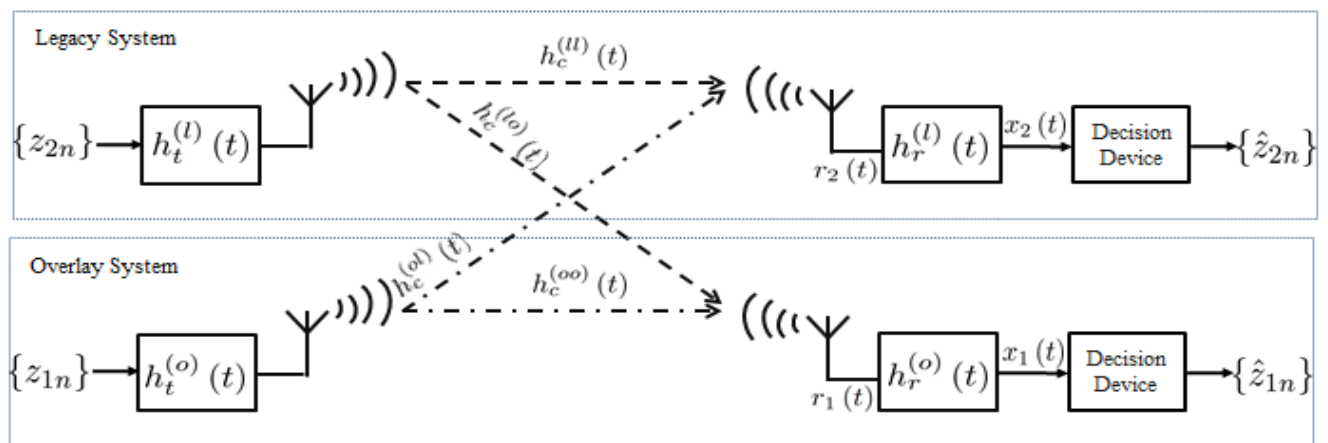

Fig. 1. System model.

The objective is to minimize the following composite MSE subject to an average power constraint, $P$, for the overlay transmitter power, $P_{t}$ (i.e. $P_{t}<P$ )

$$
M S E=M S E_{1}+M S E_{2}^{e}+\lambda\left(P_{t}-P\right)
$$

where $M S E_{1}$ represents the overlay system MSE, $M S E_{2}^{e}$ denotes the excess MSE into the legacy user, which is caused by the introduction of the overlay user, $\lambda$ counts for the Lagrange multiplier that is adjusted to satisfy the power constraint.

By transforming (1) into frequency domain, the following equations are obtained

$$
\begin{gathered}
M S E_{1}=\frac{\sigma^{2}}{T} \int_{-\frac{1}{2 T}}^{\frac{1}{2 T}}\left|H_{t}^{(o)}(f) H_{c}^{(o o)}(f) H_{r}^{(o)}(f)-T\right|^{2} d f \\
+\frac{\sigma^{2}}{T} \int_{-\frac{1}{2 T}}^{\frac{1}{2 T}}\left|H_{t}^{(l)}(f) H_{c}^{(l o)}(f) H_{r}^{(o)}(f)\right|^{2} d f \\
+N_{0} \int_{-\frac{1}{2 T}}^{\frac{1}{2 T}}\left|H_{r}^{(o)}(f)\right|^{2} d f
\end{gathered}
$$

where $T$ denotes the symbol period, $\sigma^{2}$ represents the input symbol variance, and $N_{0}$ is the additive noise level. Additionally, the first term in (2) reduces the effect of inter symbol interference, the second term suppresses the interference from the legacy system, and the last term is related to the noise power.

and

$$
\begin{gathered}
M S E_{2}^{e}=\frac{\sigma^{2}}{T} \int_{-\frac{1}{2 T}}^{\frac{1}{2 T}}\left|H_{t}^{(o)}(f) H_{c}^{(o l)}(f) H_{r}^{(l)}(f)\right|^{2} d f \\
P_{t}=\frac{\sigma^{2}}{T} \int_{-\frac{1}{2 T}}^{\frac{1}{2 T}}\left|H_{t}^{(o)}(f)\right|^{2} d f
\end{gathered}
$$

By minimizing (1) and after extensive manipulation as discussed in [8], in the case of receiver-only optimization the overlay receiver is expressed as

$$
\alpha_{2}\left|H_{r}^{(o)}(f)\right|^{4}+\alpha_{1}\left|H_{r}^{(o)}(f)\right|^{2}+\alpha_{0}=0
$$

where $\alpha_{2}, \alpha_{1}$, and $\alpha_{0}$ in (5) depend on the known channel frequency responses.
Since (5) is a quadratic function in $\left|H_{r}^{(o)}(f)\right|^{2}$, the optimal receiver can be obtained by solving for the roots of (5) as described in Algorithm 1.

In the transmitter-only optimization scenario, the overlay transmitter is given by

$$
\beta_{2}\left|H_{t}^{(o)}(f)\right|^{4}+\beta_{1}\left|H_{t}^{(o)}(f)\right|^{2}+\beta_{0}=0
$$

where $\beta_{2}, \beta_{1}$, and $\beta_{0}$ in (6) are related to the known channel frequency responses, and (6) is a quadratic function in $\left|H_{t}^{(o)}(f)\right|^{2}$. As such, the optimal transmitter can be obtained by solving for the roots of (6) using a similar approach to Algorithm 1.

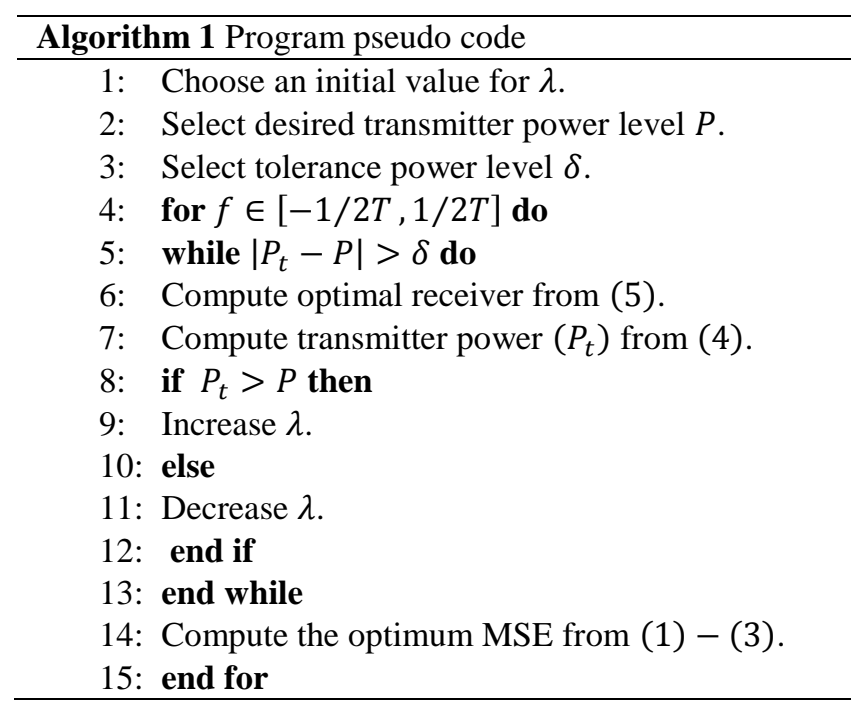

\section{ANALYTICAL AND SimULATION RESUlTS}

In this section, we provide analytical and simulation results for legacy and overlay systems operating over a flat Rayleigh fading channel corrupted by AWGN for the following scenarios:

Case 1: The overlay and the legacy users are both under strong interference. In other words, the propagation losses from the legacy system to the overlay system and from the overlay system to the legacy system are low.

Case 2: The overlay user is under stronger interference than the legacy user. In other words, the propagation loss from the legacy user to the overlay user is lower than the 
propagation loss from the overlay user to the legacy user.

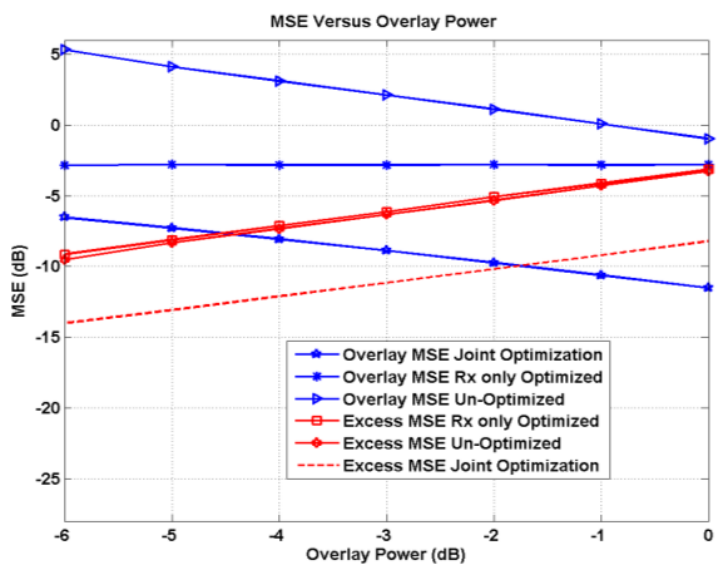

Fig. 2. The Effect of receiver-only (Rx-only) optimization on the Overlay and Legacy Systems in Case 1.

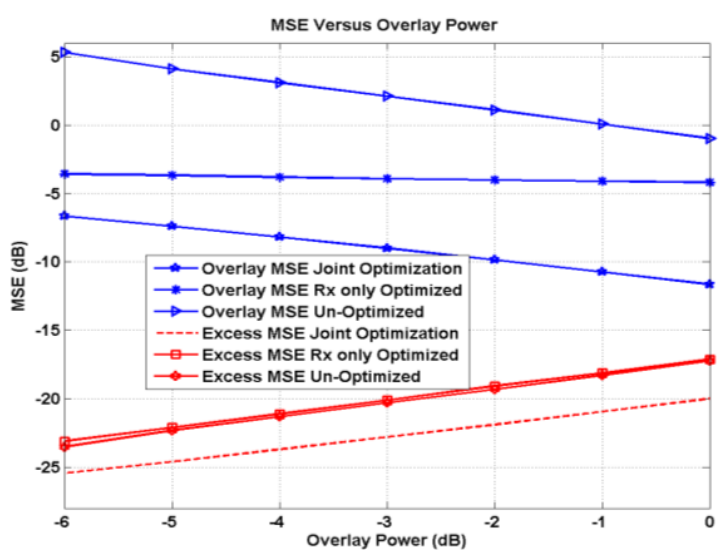

Fig. 3. The effect of receiver-only optimization on the overlay and legacy systems in Case 2.

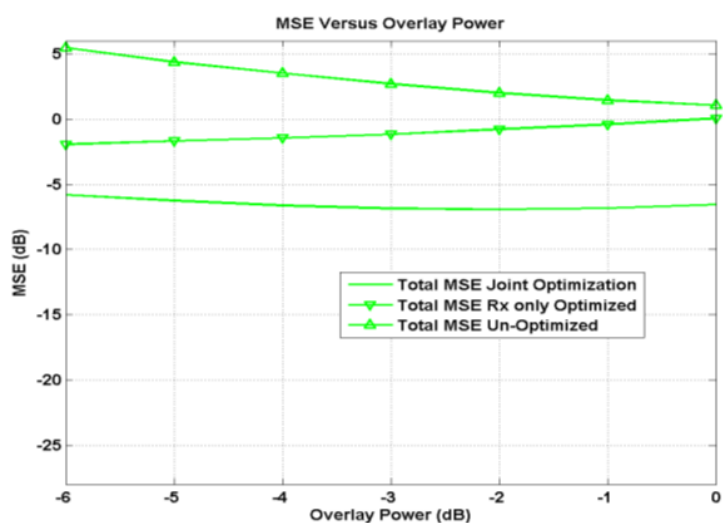

Fig. 4. Total MSE loss due to receiver-only optimization in Case 1.

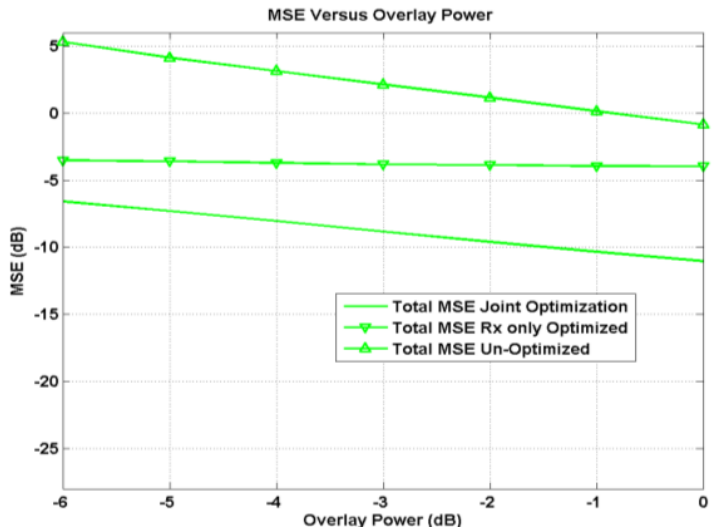

Fig. 5. Total MSE comparison loss due to receiver-only optimization in Case 2.
Without loss of generality, we assume the legacy transmitter power is fixed at $0 \mathrm{~dB}$, and the legacy and the overlay users occupy a bandwidth of $15 \mathrm{MHz}$.

The effect of optimizing only the overlay receiver on both the legacy and the overlay systems is depicted in Fig. 2 and Fig. 3 and is compared with the joint optimization of both the transmitter and the receiver of the overlay system scenario as well as the un-optimized overlay scenario, where the impact of crosstalk between overlay and legacy systems is ignored in the design of the overlay transmitter/receiver. It can be seen that the overlay system performance in the case of receiver-only optimization is between the joint optimization and the un-optimized one for the two channel cases. However, optimizing only the overlay receiver results in more degradation in the excess MSE to the legacy system.

On the other hand, Fig. 4 and Fig. 5 show the total MSE of a system with a jointly optimized transmitter/receiver overlay, a system with a receiver-optimized overlay, and an un-optimized system.

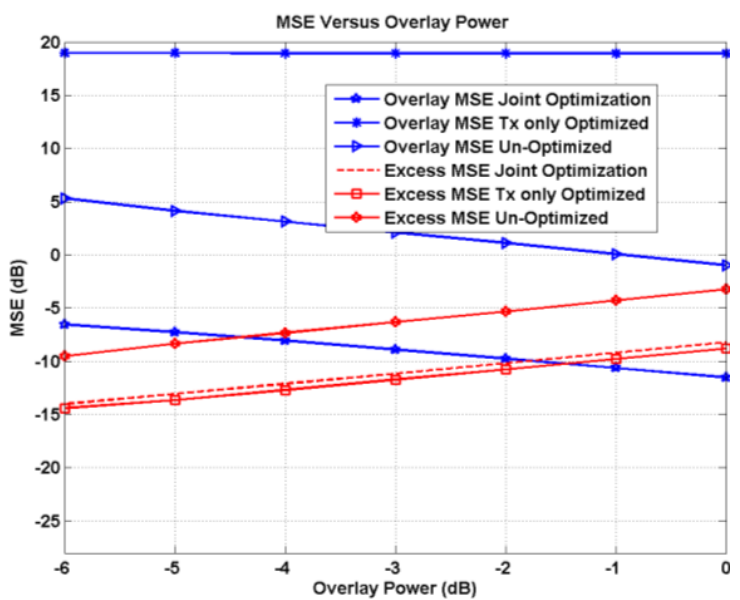

Fig. 6. The effect of transmitter-only (Tx-only) optimization on the overlay and legacy systems in Case 1.

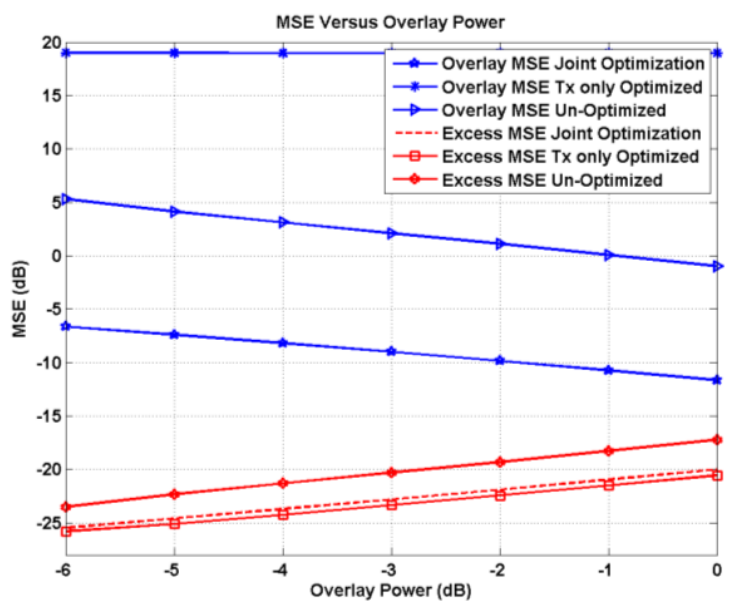

Fig. 7. The effect of transmitter-only optimization on the overlay and legacy systems in Case 2

We observe that for the two channel scenarios, in terms of the overall performance of the system (which is our cost function), the receiver-only optimization is a sub-optimum solution and for a $-3 \mathrm{~dB}$ overlay transmitter power, a total MSE loss of about $-5 \mathrm{~dB}$, compared to the joint optimized case, is incurred.

Fig. 6 to Fig. 9 compare the performance of a system with joint transmitter and receiver optimized, with a system 
having only a transmitter optimized overlay, and an un-optimized system, for the two channel scenarios. As we see, the legacy excess MSE is fairly insensitive to the transmitter-only optimization, but optimizing the overlay transmitter only leads to a significant degradation of the overlay system performance. In particular, it results in about $25 \mathrm{~dB}$ loss in the overall system performance.

In addition, by comparing Fig. 2 to Fig. 5 with Fig. 6 to Fig. 9 we can infer that the optimization of the overlay receiver has substantial effect on the system performance. That is, having an overlay system with its receiver only optimized, leads to a suboptimal performance, while reducing the complexity of the system.

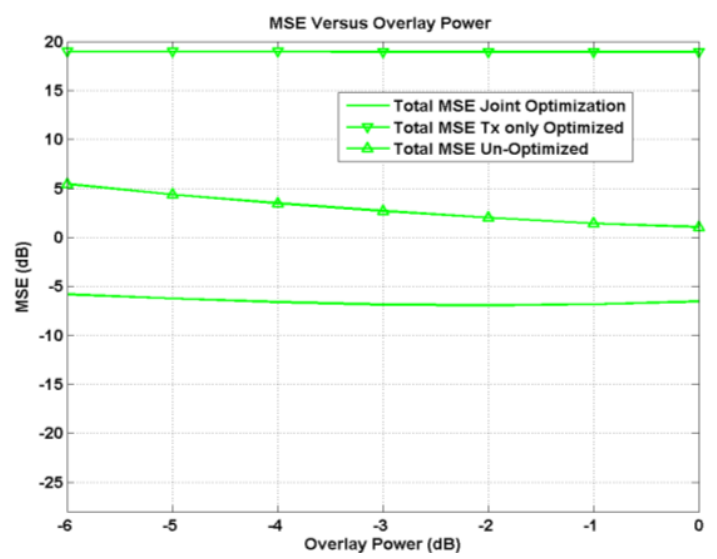

Fig. 8. Total MSE loss due to transmitter-only optimization in Case 1.

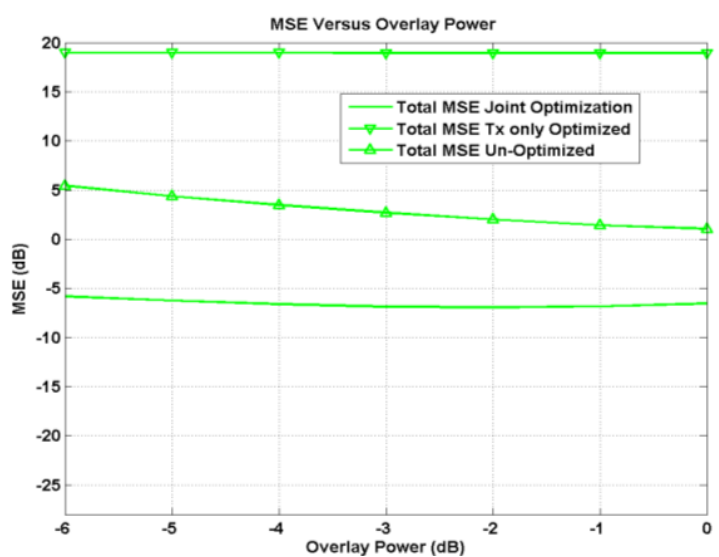

Fig. 9. Total MSE loss due to transmitter-only optimization in Case 2.

\section{CONCLUSION}

The simplified schemes for interference mitigation in overlay cognitive radio systems that are based on either optimizing the overlay user transmitter-only or the overlay user receiver-only without any changes to the existing legacy user system are presented. A composite MSE that counts for the overlay system performance while simultaneously mitigating the crosstalk introduced to the legacy user is used as a figure of merit. The analytical and simulation results under different interference scenarios show that the excess MSE to the legacy system is insensitive to the non-joint optimization. Moreover, the overall performance of a system having only an optimized receiver is between the performance of a system with jointly optimized transmitter/receiver and a system with un-optimized transmitter/receiver, while optimizing only the transmitter causes a significant degradation in the system performance.
Thus, the receiver-only optimization of the overlay system leads to a suboptimal solution.

\section{REFERENCES}

[1] Z. Zhang et al., "Self-organization paradigms and optimization approaches for cognitive radio technologies: a survey," Wireless Communications, IEEE, vol. 20, no. 2, pp. 36-42, April 2013.

[2] N. Mandayan and C. Raman, "The concept of cognitive radio," Principles of Cognitive Radio, 1st ed. Cambridge, UK, Cambridge University Press, 2013, ch. 1

[3] J. Mitola, "Cognitive Radio: making software radio more personal," Personal Communications, vol. 6, no. 4, pp. 48-52, Aug. 1999.

[4] X. Gong, A. Ishaque, G. Dartmann, and G. Ascheid, "MSEbased linear transceiver optimization in MIMO cognitive radio networks with imperfect channel knowledge," in Proc. 2010 2nd International Workshop on Cognitive Information Processing, 14-16 June 2010, pp. 105-110,

[5] L. Le and H. Ekram, "Resource allocation for spectrum underlay in cognitive radio networks," IEEE Transactions on Wireless Communications, vol. 7, no. 12, pp. 5306-5315, December 2008.

[6] B. Seo, "Joint design of precoder and receiver in cognitive radio networks using an MSE criterion,” Signal Processing, vol. 91, no. 11, pp. 2623-2629, 2011.

[7] X. Gong, M. Jordan, A. Ishaque, G. Dartmann, and G. Ascheid, "Robust MSE-Based transceiver optimization in MISO downlink cognitive radio network," in Proc. 2010 IEEE Wireless Communications and Networking Conference, 2010, pp. 1-6.

[8] M. Vameghestahbanati, H. Mir, and M. El-Tarhnui, "An MMSE overlay cognitive wireless system," International Journal of Interdisciplinary Telecommunications and Networking, vol. 4, no. 4, pp. 64-76, October-December 2012.

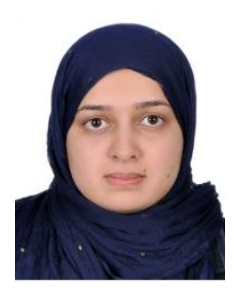

Monirosharieh Vameghestahbanati was born in Iran. She received her B.Sc. degree (summa cum laude) in electrical engineering from Ajman University of Science and Technology, Ajman, United Arab Emirates (UAE), in 2010, and her M.Sc. degree in electrical engineering from American University of Sharjah, UAE, in 2012 with the highest academic rank. She is currently working toward her Ph.D. degree in Carleton University, Ottawa, Canada.

Her research interests include iterative coding, wireless communications, and signal processing.

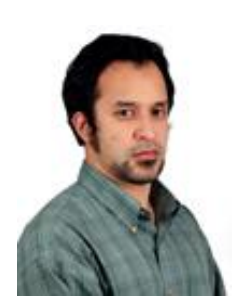

Hasan S. Mir earned a B.S. (cum laude) in 2000, an M.S. in 2001, and a Ph.D. in 2005, all in electrica engineering from the University of Washington, Seattle.

He was a member of the technical staff at the M.I.T. Lincoln Laboratory from 2005-2009. Since 2009, he has been with the Department of Electrical Engineering at the American University of Sharjah, where he is currently an associate professor.

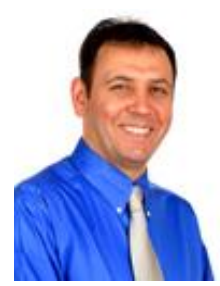

Mohamed El-Tarhuni received the B.Sc. and M.Sc degrees in electrical engineering from the University of Benghazi (formerly Garyounis University) Benghazi, Libya, in 1986 and 1990, respectively, and the Ph.D. degree in electrical engineering from Carleton University, Ottawa, ON, Canada, in 1997.

From 1987 to 1993, he was with the General Electric Company of Libya, Benghazi, Libya, as a telecommunications engineer and manager of the Communications Department. From 1997 to 2000, he was with Nortel Networks, Ottawa, ON, Canada, as a member of Scientific Staff, working on third-generation wireless communication systems using CDMA technology. Since 2000, he has been with the American University of Sharjah (AUS), Sharjah, United Arab Emirates (UAE), where he is currently a professor and the head of the Department of Electrical Engineering. From 2001 to 2006, he was the Director for the Cisco Regional Networking Academy, AUS.

Prof. El-Tarhuni has served on the organizing and technical committees of many international conferences. He served as the IEEE Industry Relations Officer for the IEEE UAE Section from 2002 to 2006. 